Shows the association with the $3 \mathrm{M}$ Corporation Severity of Index score with CAHR-AT Score $\geq 5$.

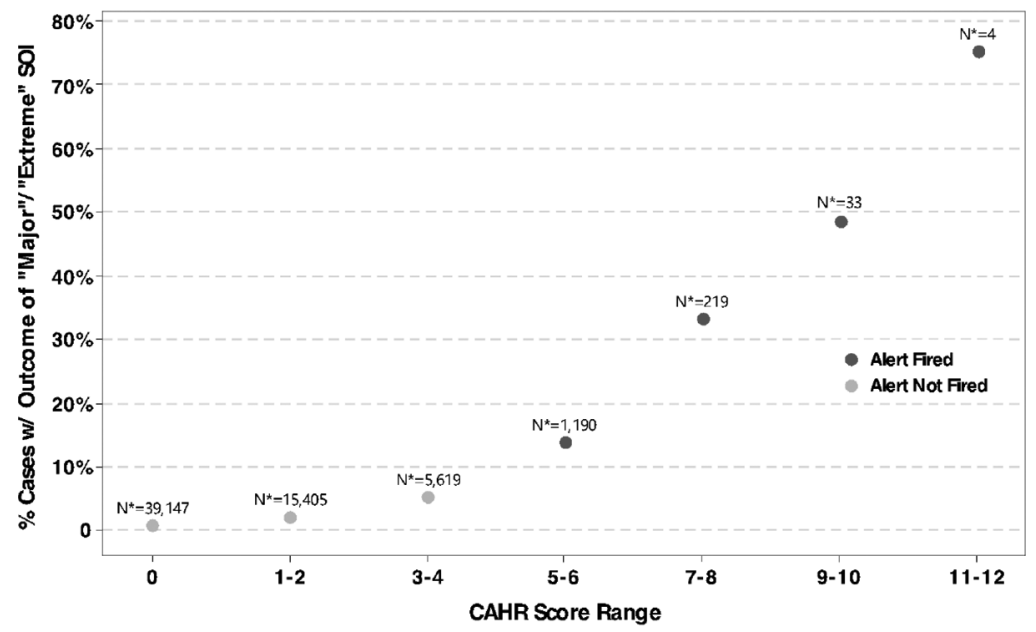

Based on 61,617 Cases During 11/12 - 12/12, 08/14 - 01/15, and 01/17-06/17

* Number of cases with the indicated CAHR score range

Abstract 1 Figure 5 Association of CAHR-AT score and severity of illness

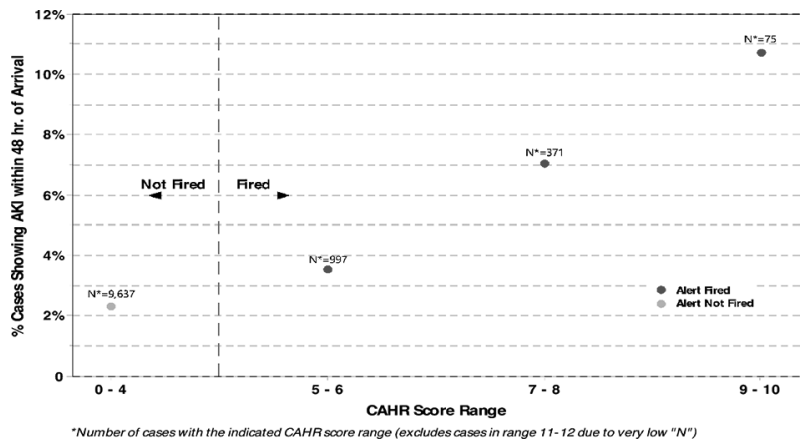

Abstract 1 Figure 6 Association of CAHR-AT score with incidence of acute kidney injury (AKI) among admitted patients within $48 \mathrm{hrs}$ of arrival

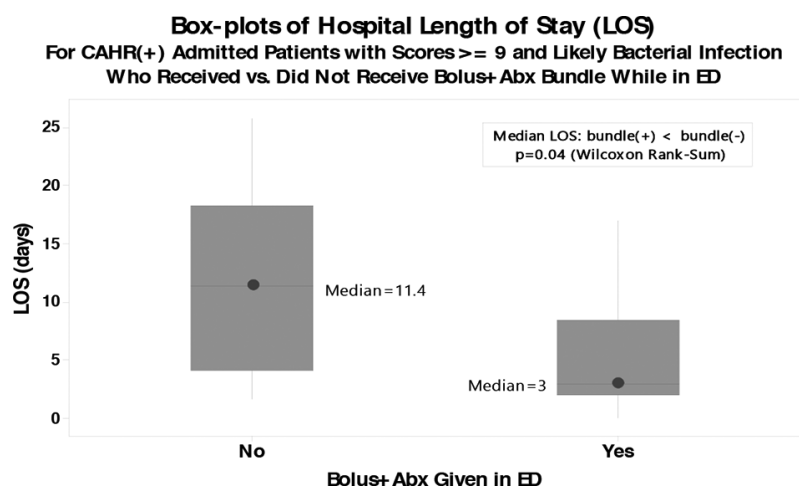

Abstract 1 Figure 7 Differences in length of stay (LOS in days) between CAHR+ patients who received the bundle (IVF and IV antibiotics) vs. those who did not

Objectives This stand-alone urban children's hospital ED aims to improve team-based care, situational awareness, and patient outcomes through team huddles and associated interventions (figure 1).

Methods Development of the CAHR-AT utilized vital signs data of $>1 \times 10^{\wedge} 6$ patients to derive standards. Logistic regression and 'machine-learning(AI)' identified factors showing the highest association with gold-standard sepsis cases and applied weights to each factor for optimum sensitivity. A nursing assessment form was added to the dyad assessment process and visual redesign of the tool interface went into effect using a stoplight approach with red, yellow, and green lights indicating patient acuity and resources needed (figure 2).

Results It has been over 239 days (934 alerts) since the last unanswered alert by the provider/nurse dyad (figure 3). The average percent of CAHR patients with a completed initial huddle increased from $9.3 \%$ to $45.3 \%$ (figure 4). Higher CAHR-AT scores were associated with higher severity-ofindex (SOI) and acute kidney injury (AKI) within $48 \mathrm{hrs}$ of arrival (figures 5 and 6). Preliminary data show CAHR-AT patients with a score $\geq 8$ who received the bundle (IV-fluid bolus and IV-antibiotics) significantly shorter length of stays (figure 7).

Conclusions CAHR-AT predicts physiologic decompensation and AKI. Its processes promote team-based critical thinking and improve patient outcomes. Next steps include prescriptive order sets for both red/yellow stoplight activations and spread to inpatient units.

\section{IMPROVING THE TIMELINESS OF CARE FOR CHILDREN WITH TESTICULAR TORSION IN THE PEDIATRIC EMERGENCY DEPARTMENT}

${ }^{1}$ Sri Chinta, ${ }^{2}$ Matthew Gray, ${ }^{2}$ Matthew Kopetsky, ${ }^{3}$ Shannon Baumer-Mouradian, ${ }^{3}$ Amy Drendel, ${ }^{3}$ Elizabeth Roth, ${ }^{3}$ Catherine Ferguson, ${ }^{3}$ Kevin Boyd, ${ }^{3}$ David Brousseau. ${ }^{1}$ Medical College of Wisconsin/Children's Hospital of Wisconsi, USA; ${ }^{2}$ Children's Hospital of Wisconsin, USA; ${ }^{3}$ Medical College of Wisconsin, USA

\subsection{6/bmjoq-2019-ihi.2}

Background Early diagnosis and timely surgery are critical to treat children with testicular torsion. Only 33\% of patients met the hospital goal of 'critical diagnosis to operating room time of 60 minutes or less [CDOR60]' prior to starting the QI project. 


\section{Abstract 2 Figure 1}

Testicular Pain evaluation Simplified Failure Mode Effects Analysis (sFMEAC)

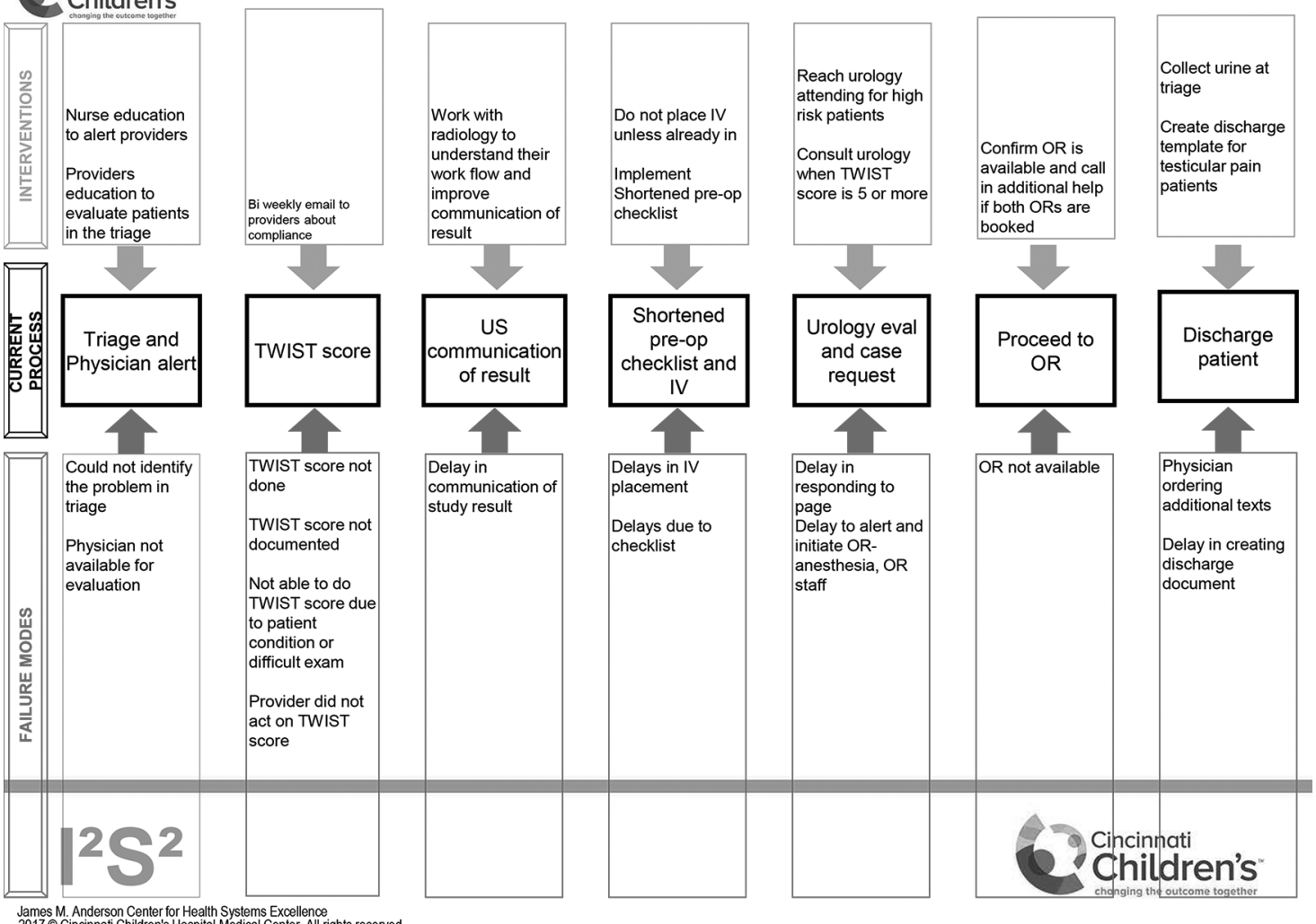

axy chininati

Project Leader(s): sri s chinta

\section{Improving The Care Of Children With Testicular Pain Key Driver Diagram (KDD)}

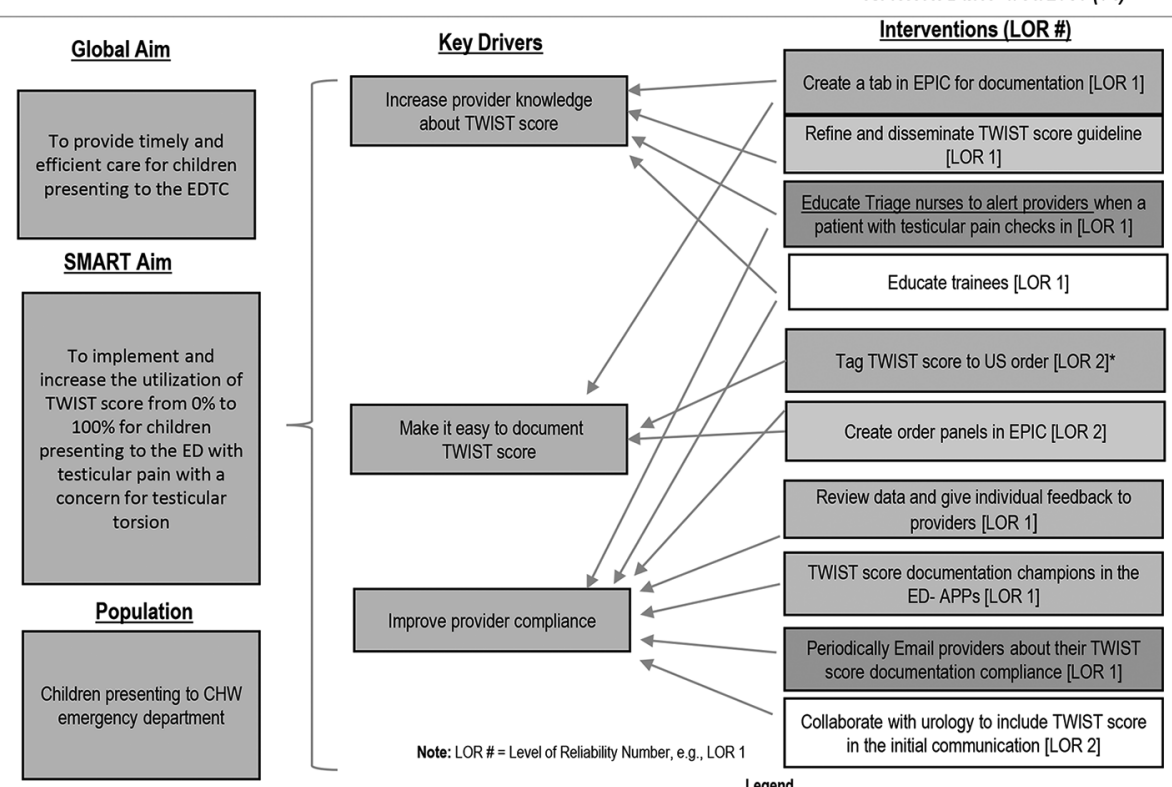

Legend

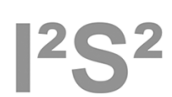


Cincinnati

\section{TWIST score utilization rate}

Percentage of patients with TWIST score documented biweekly

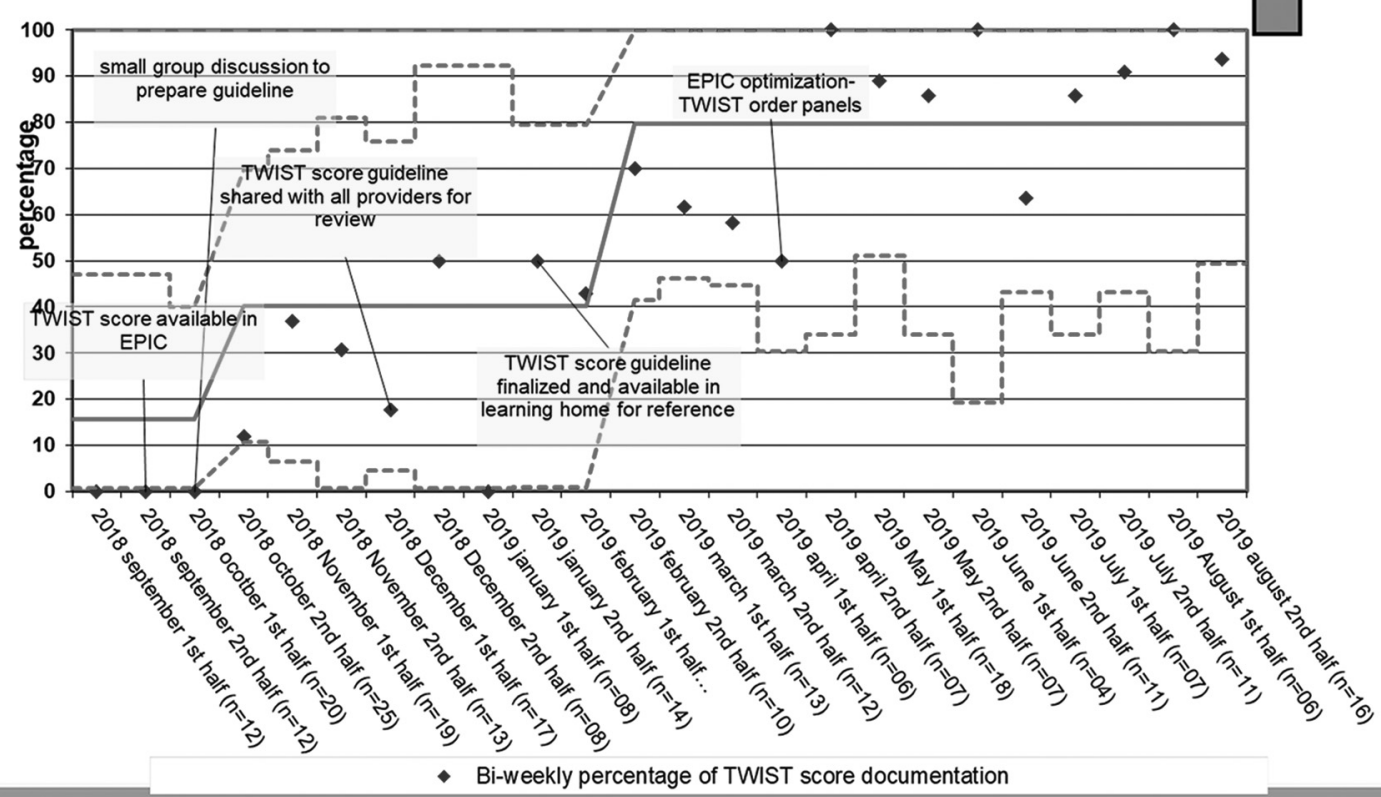

\section{$\|^{2} S^{2}$}

\section{Abstract 2 Figure 3}

Sy Cincinnati
Children's

US critical result to OR time 2018 January to 2019 August

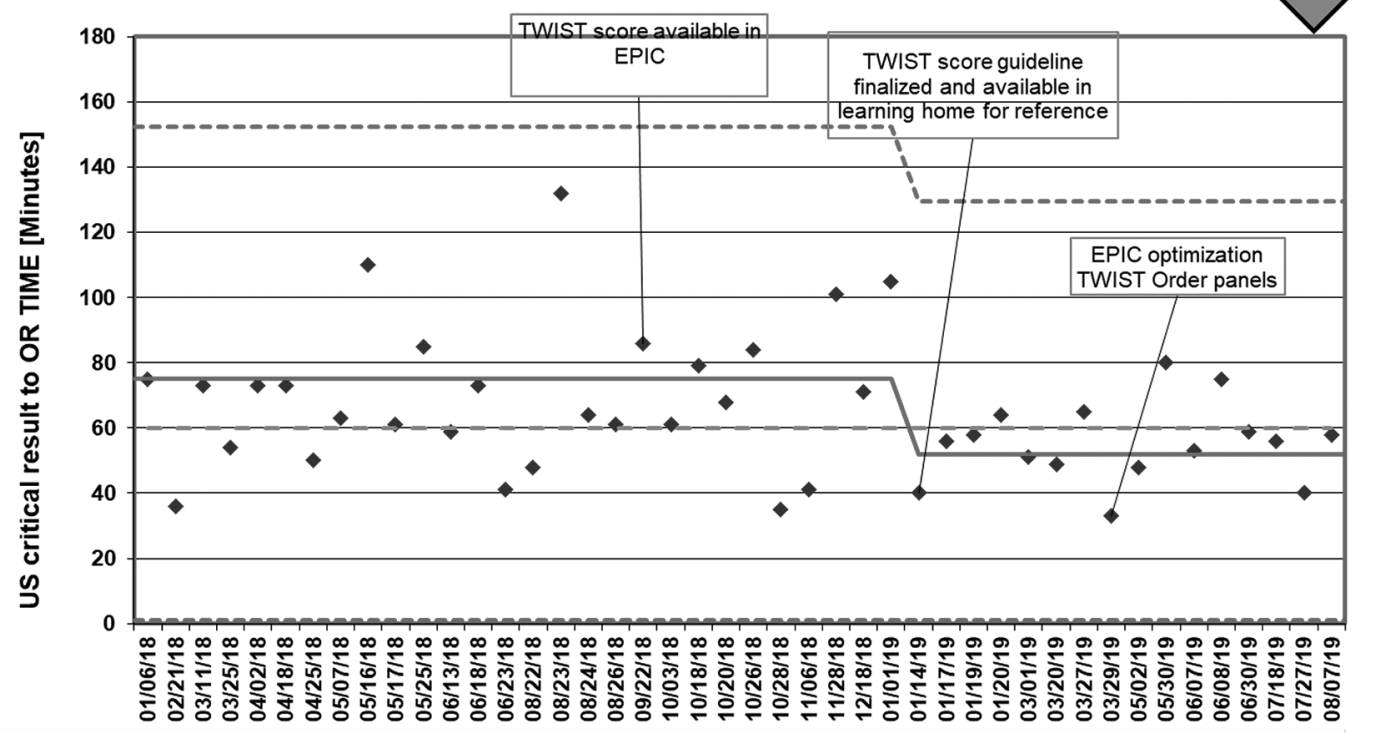

- Individual patient

- Average time US critical result to OR

--- Control Limits 
Objectives The objective of the quality improvement project was to increase the utilization of TWIST score, a validated clinical scoring system from $0 \%$ to $80 \%$ over 12 months period for children evaluated for testicular torsion, as a means of increasing the number of patients with CDOR60.

Methods Deploying the Institute for Healthcare Improvement Model for Improvement, we formulated an aim statement and identified key drivers. Of our interventions, successful implementation of the TWIST score in the emergency department (ED), with a guideline to support earlier notification of urology for high risk patients with testicular pain, was determined to be the highest impact intervention. We educated providers and gave biweekly feedback about guideline adherence, created order panels and documentation tabs in our electronic medical record to encourage documentation.

Results Between September 2018 and April 2019, TWIST score documentation improved to $>80 \%$ and was sustained at that rate for 4 months. After implementation of the guideline, the critical diagnosis to OR time of 60 minutes or less was achieved in 12 out of 16 patients diagnosed with testicular torsion in the ED [75\%] compared to our baseline of $33 \%$.

Conclusions A quality improvement project to improve the timeliness of care for children with testicular torsion resulted in delivery of expedited surgical care for these patients.

\section{PLANNED COMMUNITY PARAMEDICINE VISIT PROGRAM REDUCE ED VISITS AND READMISSIONS}

${ }^{1}$ Amanda Ascher, ${ }^{2}$ Hanan Cohen, ${ }^{3}$ Zoë Stopak-Behr, ${ }^{4}$ Irene Kaufmann. ${ }^{1}$ Bronx Partners for Healthy Communities, SBH Health, USA; ${ }^{2}$ Empress, USA; ${ }^{3}$ SBH Health System, USA; ${ }^{4} B r o n x$ Partners for Healthy Communities, SBH Health, USA
Background Many patients with multiple chronic conditions have challenges in understanding, accessing and navigating the healthcare system, leading to frequent ED use, admissions and readmissions. We piloted a planned Community Paramedicine Visit Program (CPVP) to patients at risk for repeated ED visits or admissions, in an effort to reduce hospital utilization in a community safety-net hospital.

Objectives Reduce avoidable hospital use by identifying highrisk patients with patterns of high utilization and referring them to CPVP.

Methods This pilot was conducted in The Bronx, NY with a single EMS agency and a single safety-net hospital, focused on Medicaid patients as part of a NYS Medicaid waiver. Initial challenges of identifying and referring high-risk patients were addressed using PDSA ramps. Analysis looked at hospital use for each of the 43 unique patients referred into the CPVP and compared equal time periods pre- and post-referral for each patient. The analysis does not include patients who refused referral or accepted referral but refused enrollment into CPVP.

Results The cohort had 160 visits to the hospital (inclusive of emergency department visits, admissions and readmissions) in the 1 to 6 months prior to their referral. The same cohort of patients had only 65 hospital visits in the 1 to 6 months after their engagement in the CPVP; this represents a $59.4 \%$ reduction in hospital use and a $59.4 \%$ decrease in the average number of visits per patient.

Conclusions The Community Paramedicine Visit Program decreased hospital use by high-risk patients. Team-based QI efforts help engage more patients in this valuable program.

10.1136/bmjoq-2019-ihi.3

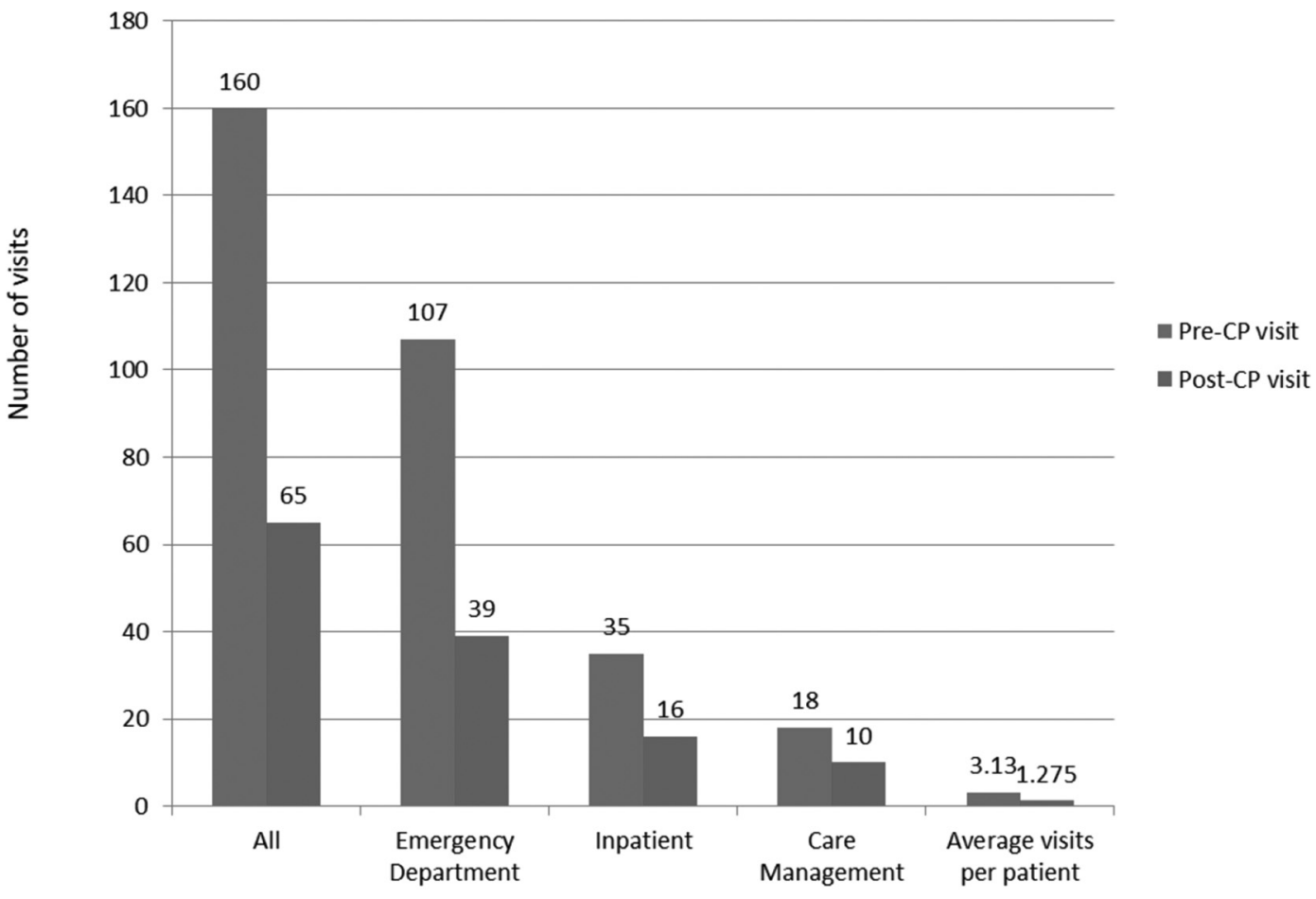

Referral Source 